This item was submitted to Loughborough's Research Repository by the author.

Items in Figshare are protected by copyright, with all rights reserved, unless otherwise indicated.

\title{
Managing loads on aircraft generators to prevent overheat in-flight
}

PLEASE CITE THE PUBLISHED VERSION

http://dx.doi.org/10.4271/2014-01-2195

\section{PUBLISHER}

(C) SAE International

\section{VERSION}

SMUR (Submitted Manuscript Under Review)

\section{PUBLISHER STATEMENT}

This work is made available according to the conditions of the Creative Commons Attribution-NonCommercialNoDerivatives 4.0 International (CC BY-NC-ND 4.0) licence. Full details of this licence are available at: https://creativecommons.org/licenses/by-nc-nd/4.0/

\section{LICENCE}

CC BY-NC-ND 4.0

\section{REPOSITORY RECORD}

Graham, James H., Roger Dixon, Peter D. Hubbard, and lan Harrington. 2019. "Managing Loads on Aircraft Generators to Prevent Overheat In-flight". figshare. https://hdl.handle.net/2134/18953. 


\title{
Thermal Modelling of an Alternator for Use in a Prediction System
}

\author{
James Graham, Roger Dixon, Peter Hubbard \\ School of Electronic, Electrical and Systems Engineering, Loughborough University, \\ Loughborough, Leicestershire, LE11 3TU \\ Contact: J.H.Graham@lboro.ac.uk
}

\begin{abstract}
On future UAVs it is envisaged that the power requirements of all on-board electrical systems will increase. Whilst, in most flight (mission) situations the installed generation capacity will have adequate capacity to operate the systems, it is possible that during certain abnormal situations the generators on-board may be forced to operate under very high load conditions. The main failure mechanism for a generator is overheating and subsequent disintegration of windings, hence the research problem being addressed here is that of modeling the thermal dynamics of a generator in such a way that the model can be used to predict future temperatures given knowledge of the future mission requirements. The temperature predictions will be used to allow prioratising of the mission actions in order to get the most out of a generator without overheating it.

The research presented here summarises the modeling of the generator and formation of the load management system. Results are presented showing the system reallocating loads after a fault during flight, preventing overheat of the generators and successfully completing the mission.
\end{abstract}

\section{INTRODUCTION}

The current trend of increased use of electrical power to run systems on-board an aircraft [1] [2] has lead to much research being undertaken to improve system performance, reliability and maintainability. Flight critical systems previously operated by other means, such as hydraulics or pneumatics, now need to be guaranteed a constant supply of electricity to be considered safe. This aim of this study is to look at power supply within aircraft, focusing particularly on power generation, aiming to develop methods of ensuring more reliable supply of power. The ability of a generator to supply power is based upon its thermal state assuming there are no other faults on the system. Under normal circumstances a well designed generator will easily operate within its thermal limits, but certain abnormal situations, for example a blockage in coolant flow may force generator temperature to rise which may in turn heat the generator above its thermal limits.

The main failure mechanism for a generator is overheating and subsequent disintegration of windings, hence the research problem being addressed here is that of modeling the thermal dynamics of a generator in such a way that the model can be used to predict future temperatures given knowledge of the future mission requirements. The temperature predictions will be used to allow prioratising of the mission actions in order to allow maximum utilisation of power generation capacity without overheating.
Current trends in aircraft health management are more focused towards maintenance, examples include Iyer et. al. [3] and Hess et. al. [4]. However the work described here is focused upon on-board health management, systems which focus on reconfiguration during flight. Rouet et.al. [5] have looked at real-time prognostics performed while the aircraft is in flight. However this paper particularly addresses the hardware side of the problem, with little information given regarding the PHM process itself. Tang et. al. [6] however do look at on board support, and in-flight reconfiguration for engines. Of the research found on aircraft generators the work was still focused upon maintenance, examples include Watson et. al. [7] and Batzel and Swanson [8].

The method proposed here to manage the loads on the generator will be based upon the flow diagram shown in figure 1. The loop repeats periodically over the length of the flight constantly checking the generator to ensure the current load plan doesn't cause an overheat.

Within each cycle there are three main stages:

1) State Update

2) Prediction

3) Optimisation

The state update phase is where current and previously recorded data is used to update the states within the model so that they match the real system as closely as possible. The model is then used to predict the effect of the current load profile upon the temperature of the generator during the prediction phase. Finally if an overheat error is detected the system then re-optimises the load profiles to prevent overheat while still allowing mission completion.

Using this method the profile is constantly checked for problems, this includes re-checking and if necessary re-optimising the load profile if predictions a long period into the future are not accurate enough. This mean that when the time comes to actually implement the profile upon the generator the solution will be correct and not cause an overheat.

The rest of this paper describes the research undertaken to achieve the aims stated above. Section II. describes the modeling process and prediction section III. the derivation of a simplified version of the model. Section IV. describes the load management system and presents results. Finally section V. concludes the paper. 


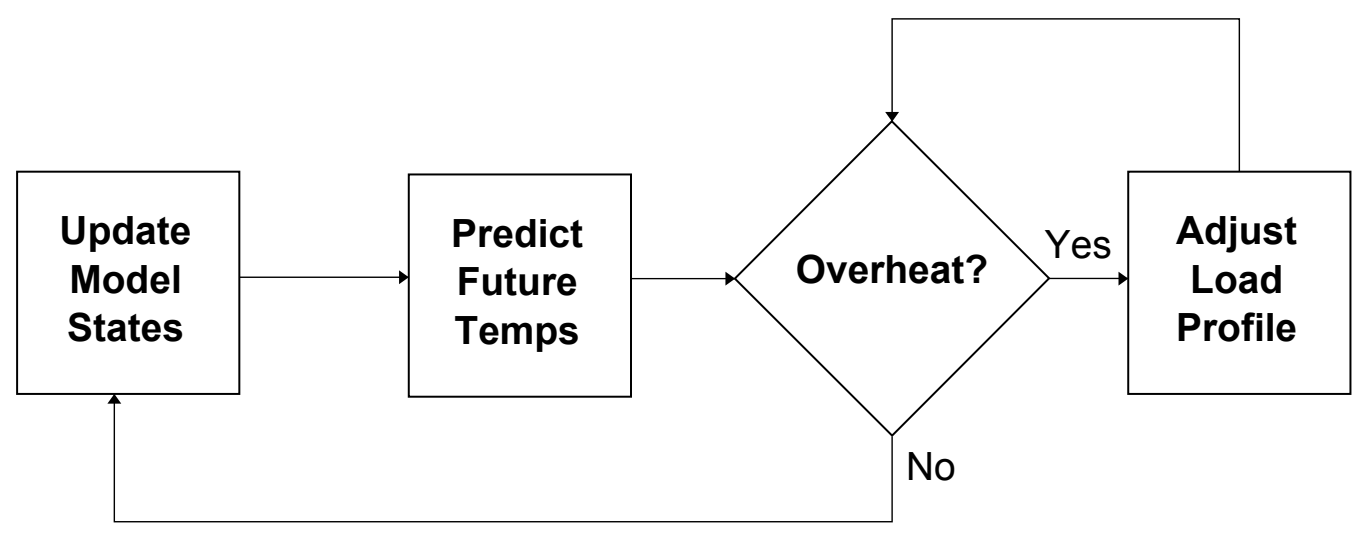

Fig. 1. Flow diagram of Load Management System.

\section{Thermal Modeling AND Prediction}

\section{A. Model}

Previously a paper has has been published which discussed in detail the thermal modeling process [9], however the model used will be summarised here.

The method used to model the generator is a thermal network model, chosen for the fact that it has low computational requirements while still maintaining good accuracy. Extra information on this type of model can be found in the research of Mellor et al. [10] and Perez and Kassakian [11].

The basic cylindrical structure (figure 2) on which all the metal sections of the generator is base upon models the heat transfer through the section in two directions; radial and axial. This is achieved with two thermal resistance networks. A third thermal resistance is then connected to each relating the networks to the average temperature of the whole cylinder. This allows the two networks to be connected forming the thermal model for the whole cylinder, and giving the point at which heat it is assumed that the axial temperature in the components of the model is constant, this is shown in figure 3 as a single thermal resistance $R_{a}$ representing the flow of heat from the mean temperature to the ends of the cylinder. The equations for the network are shown below.

$$
\begin{aligned}
R_{a} & =\frac{L}{12 \pi k_{a}\left(r_{1}^{2}-r_{2}^{2}\right)} \\
R_{r 1} & =\frac{1}{4 \pi k_{r} L s}\left(1-\frac{2 r_{2}^{2} \log \left(\frac{r_{1}}{r_{2}}\right)}{r_{1}^{2}-r_{2}^{2}}\right) \\
R_{r 2} & =\frac{1}{4 \pi k_{r} L s}\left(\frac{2 r_{1}^{2} \log \left(\frac{r_{1}}{r_{2}}\right)}{r_{1}^{2}-r_{2}^{2}}-1\right) \\
R_{r 3} & =\frac{1}{8 \pi k_{r} L s\left(r_{1}^{2}-r_{2}^{2}\right)}\left(r_{1}^{2}+r_{2}^{2}-\frac{4 r_{1}^{2} r_{2}^{2} \log \left(\frac{r_{1}}{r_{2}}\right)}{r_{1}^{2}-r_{2}^{2}}\right)
\end{aligned}
$$

Where $R_{a, r 1, r 2, r 3}$ are the resistance shown in figure 3 . From figure 2, $L$ is the cylinder length, $r_{1}$ and $r 2$ are the outer and inner radius respectively. Finally $k_{r}$ and $k_{a}$ are the axial and radial thermal conductances, and $s$ is the stacking factor.

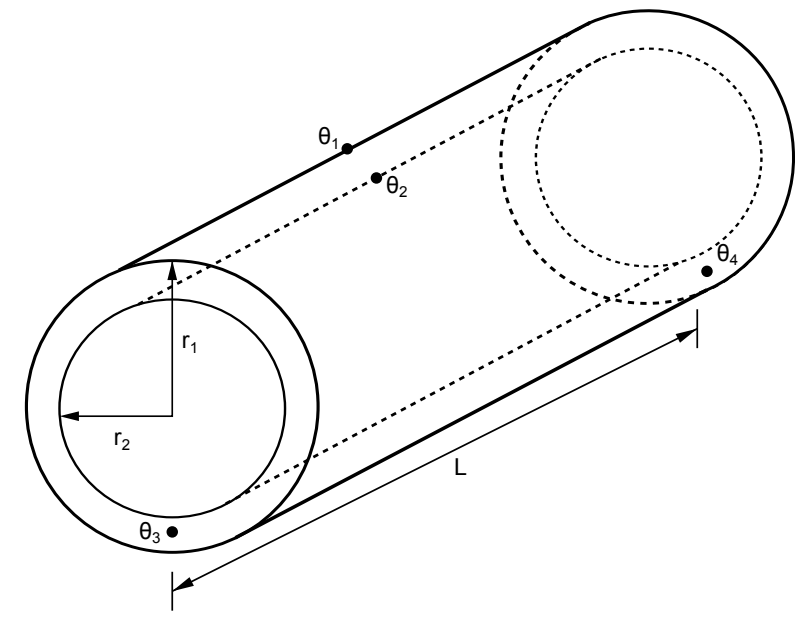

Fig. 2. Cylindrical component [10].

Figure 3 also shows the heat input to the cylinder in Watts as a constant current source, and shows the heat storage of the cylinder as a capacitance. The heat storage of the cylinder is calculated using equation 5 .

$$
C=\rho c_{p} \pi\left(r_{1}^{2}-r_{2}^{2}\right) L
$$

\section{B. Model Solution}

Figure 4 shows the model divisions with the exception of the stator end windings and the air in and out. A model for each of these divisions is derived, they are then linked together to form the full model.

With the full network defined a nodal analysis can be undertaken on the full thermal circuit to define the equations that need to be solved to calculate the temperatures over time. Equations 6 and 7 show the nodal equations that are derived from the standard cylindrical component, in this case for the stator iron. 


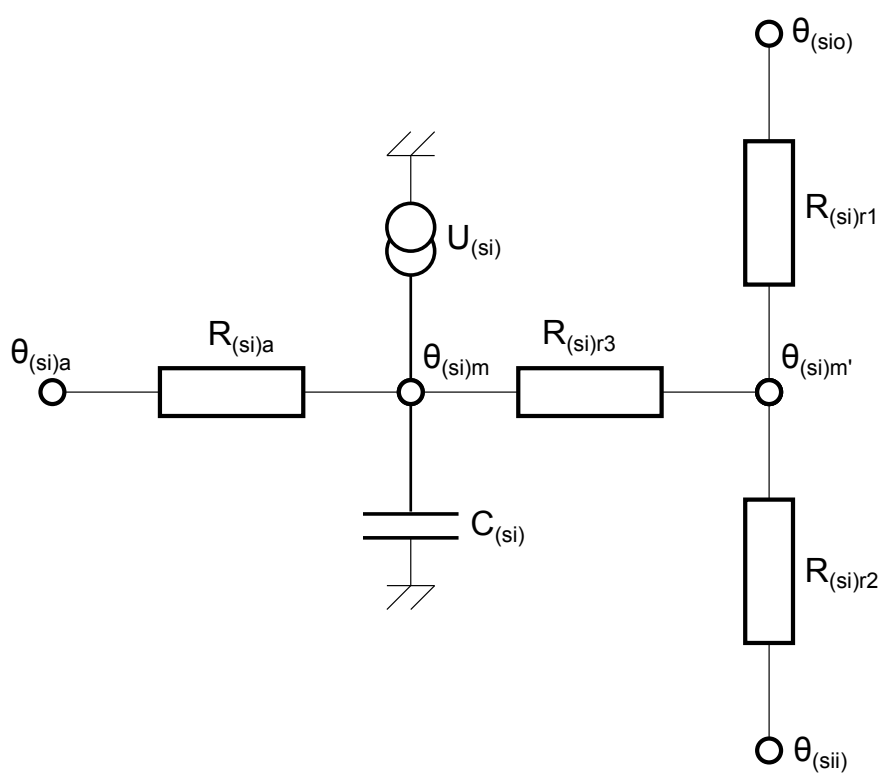

Fig. 3. Thermal Network for cylindrical component.

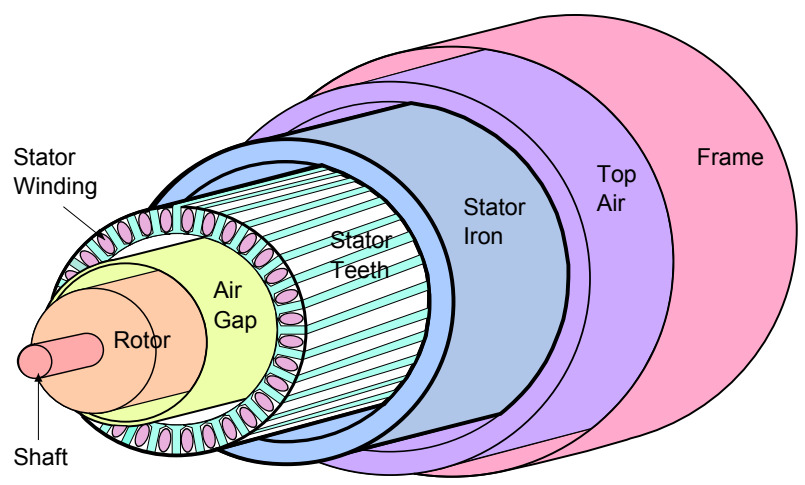

Fig. 4. Model divisons.

$$
\begin{aligned}
C_{\text {si }} \frac{d \theta_{\text {sim }}}{d t}= & \frac{1}{R_{\text {sia }}}\left(\theta_{\text {sia }}-\theta_{\text {sim }}\right) \\
& +\frac{1}{R_{\text {sir } 3}}\left(\theta_{\text {sim }}-\theta_{\text {sim }}\right)+U_{\text {si }} \\
0= & \frac{1}{R_{\text {sir } 1}}\left(\theta_{\text {sio }}-\theta_{\text {sim }}\right) \\
& +\frac{1}{R_{\text {sir } 2}}\left(\theta_{\text {sii }}-\theta_{\text {sim }}\right)+\frac{1}{R_{\text {sir } 3}}\left(\theta_{\text {sim }}-\theta_{\text {sim }}\right)
\end{aligned}
$$

Where the subscript si denotes that the value is from the stator back iron, with the letters $a$ and $r$, denoting axial and radial directions respectively, $R_{\text {sia }}$ for example is the stator iron axial resistance. $C_{s i}$ is the heat storage for the stator back iron, $\theta_{s i m}$ is the average temperature of the cylinder, $U_{s i}$ is the heat input to the stator iron. $\theta_{\text {sia }}$ and $\theta_{\text {sim }}$ are the temperatures at the adjacent nodes, with $R_{\text {sia }}$ and $R_{\text {sir } 3}$ being the thermal resistance between them.

Each node can be expressed in a similar manner to equation 6 and 7, these equations can then be expressed in matrix form

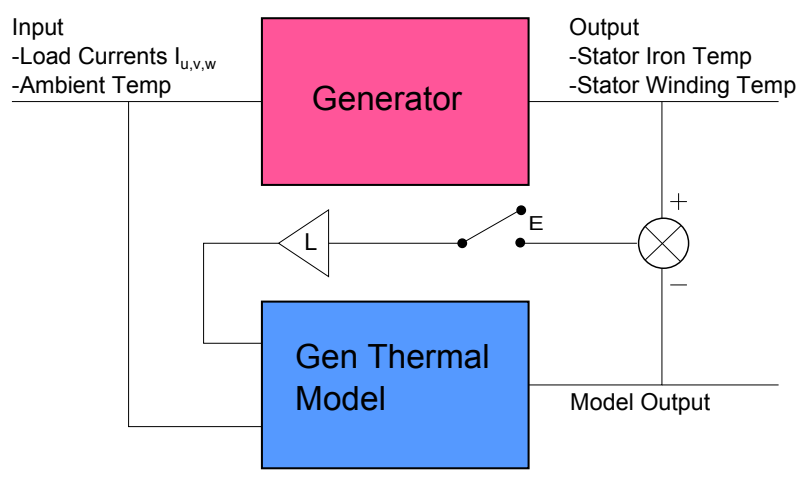

Fig. 5. N-step ahead prediction using Kalman filter with gain L.

$$
[C] \frac{d[\theta]}{d t}=[G][\theta]+[u]
$$

where $[C]$ is a square matrix of thermal heat storage values, $[G]$ is a square matrix of thermal conductance between nodes, $[\theta]$ is the node temperature states, and $[u]$ is a column matrix of heat sources. The above differential equations allow the network to be solved for transient conditions.

The initial nodal analysis gives 19 equations, but a large number of these are intermediary nodes or are air nodes where $C$ is assumed to be zero. These can be mathematically eliminated to leave only the 8 equations.

\section{Kalman Filter}

The model discussed in the previous section is used as part of a Kalman Filter to complete the state update sub-system. The Kalman filter was implemented on a discrete time version of the model with known Q and R matricies, and overview of this can be found in the paer by Welsh and Bishop [12].

The Kalman Filter completes the state update by reducing the error between the model and the real system to almost zero. This means that the prediction made by the model on its own starts from the correct temperature states.

\section{Prediction}

As shown by Brown and Hwang [13] the Kalman filter can be used for n-step ahead prediction. The method shown in figure 5, shows that while switch $\mathrm{E}$ is closed the Kalman filter is active and measurement data is used to update the model. When $\mathrm{E}$ is open the model is run in open loop mode, which is used to make predictions.

\section{SIMPLIFIED MODEL}

When testing the final load management system it was necessary to create a version of the model that was simpler than the model created previously. This would be used in simulation tests along side the more complex version to test the load management system.

The model was simplified by linearising the calculation of the winding losses In the full model this is calculated by using the following: 


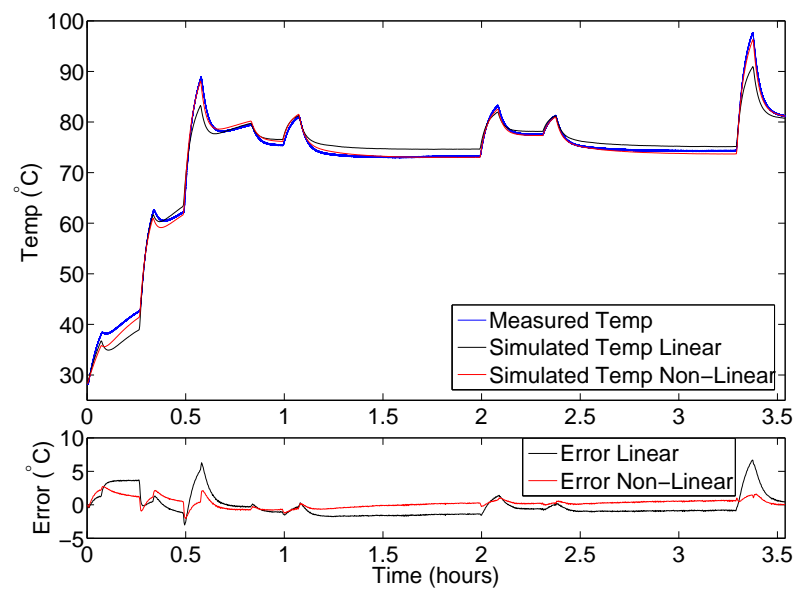

Fig. 6. Comparison of Winding Temperature of the Linear and Non-Linear models to Actual Generators.

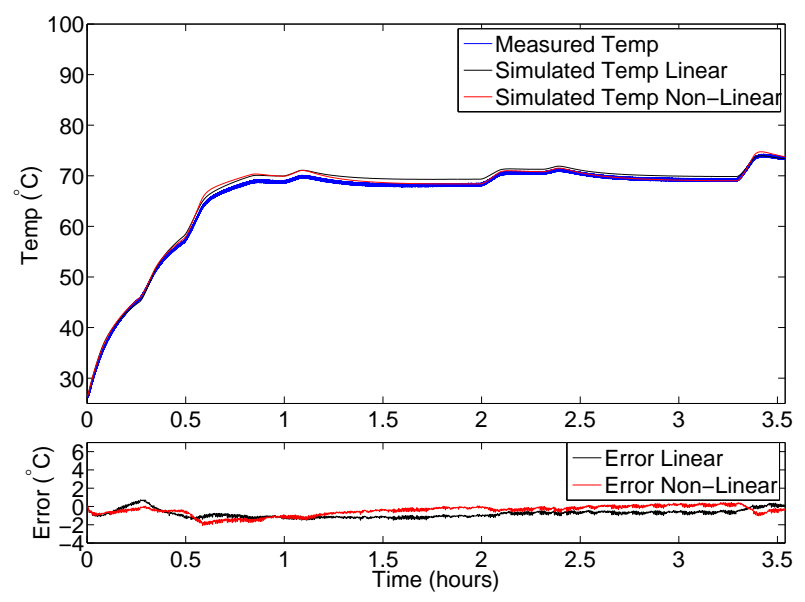

Fig. 7. Comparison of Stator Iron Temperature of the Linear and Non-Linear models to Actual Generators..

$$
P_{c u, v, w}=I_{u, v, w}^{2} R_{s w u, v, w}
$$

Where $P_{c}$ is the loss in the $\mathrm{u}, \mathrm{v}$ or $\mathrm{w}$ winding, $I_{u, v, w}$ is the current in the $\mathrm{u}, \mathrm{v}$ or $\mathrm{w}$ windings and $R_{s w u, v, w}$ is the resistance of the $\mathrm{u}, \mathrm{v}$ or $\mathrm{w}$ winding. The power loss for the three windings is then added together for the total loss.

To simplify the model the $I_{u, v, w}^{2}$ term was linearised over the range of the generator, in this case 0 to $7 A$. In addition due to the generator being used in balanced operation the winding loss for a single phase was calculated then multiplied by three. Figures 6 and 7 below shows results comparing the simplified model to the actual test rig and to the more complex model.

Comparing, the two models both perform well in respect to the shape of the curve. However the non-linear model has greater agreement with the data from the generator. The linear model has a greater steady state error as well as greater errors during transient operation, however the accuracy is sufficient for use in the load management system as part of the Kalman Filter and for prediction.

\section{LOAD MANAGEMENT}

The final stage shown in figure 1 is the load adjustment, this stage is the re-optimisation of the load profile applied to the generator over time to prevent any overheat failures. The design of this system is such that after each check the load can be optimised, this includes re-optimisation if for example other errors happen or predictions made far into the future were not accurate enough.

Due to the way in which the various actions by the aircraft are undertaken, in order to complete an action (e.g. surveillance) the power required for all the flight systems, plus the extra for any specific action must be available for the duration of the action for it to be completed. In reality this means that at any time the aircraft is in one of two discrete states, either completing an action or resting in a power saving mode to cool the generators.

This lead to the load optimisation aspect to be defined in terms of time. In this case the total flight time is defined as the sum of the time required to complete the actions for the mission, plus any additional rest time. The aim is then to minimise the flight time as part of the optimisation under the constraint that the generators on-board must not overheat. This can be represented as:

$$
\begin{array}{ll}
\text { Minimise } & T_{m}=\sum_{k=1}^{n} T(k)+\sum_{r=1}^{n} T(r) \\
\text { Subject to } & \theta_{s i, s w}(t) \leq \theta_{\max } \\
& 0 \leq t \leq T_{m}
\end{array}
$$

Where $T_{m}$ is the total length of time it takes to complete the mission, $k$ references the different actions that need to be completed in order to finish the mission, $r$ references the rest periods, with $T(k, r)$ being the length of time required for the action referenced by $k$ or $r . \theta_{s i, s w}(t)$ is the temperature of the stator iron and winding at time $t$ in the mission defined by equation 12 and $\theta_{\max }$ being the maximum temperature of the generator.

When choosing the method for optimisation the discrete nature of the problem defined that at any point the aircraft would be executing a mission action in its entirety or it would be resting. This lead to two options for optimising the load which are detailed below. These are generator resting and load displacement where multiple generators are available.

\section{A. Generator Resting}

While a generator can not be switched off in flight as a minimum power needs to be supplied to keep the aircraft flying, it is possible to switch off all non-necessary loads running just the minimum required to keep aloft. This creates a minimum power mode and while in this mode less power is used than in any other mode, the effect of this is to cool the generator. This forms the basis of this load management method. 
If an overheat is sensed the optimiser finds the minimum time required in rest mode that will allow the action that caused the overheat to be completed. This can be defined as:

$$
\begin{array}{ll}
\text { Minimise } & T_{r} \\
\text { Subject to } & \theta_{s i, s w}(t) \leq \theta_{\max } \\
& 0 \leq t \leq T_{r}
\end{array}
$$

\section{B. Load Displacement}

Load displacement can only be used on an aircraft where multiple generators are present. This process involved using any spare capacity available in secondary generators to lower the loads seen by the faulty generator to the point that an overheat no longer occurs. This can be summarised as:

$$
\begin{array}{ll}
\text { Minimise } & I_{d} \\
\text { Subject to } & \theta_{s i, s w}(t) \leq \theta_{\max } \\
& 0 \leq t \leq T_{k}
\end{array}
$$

\section{Validation}

In order to test the final system the two versions of the thermal model described earlier were used to create a test which would show both successful optimisation of the load to prevent overheat and also show robustness in the system. The test setup involved simulating an aircraft with two generators completing a mission with a fault occurring on one part way through the test. To be successful the system would have to both prevent overheat and allow completion of the mission. The temperature limit set for this test is $100^{\circ} \mathrm{C}$.

To generate these load profiles a set of various actions that can be undertaken by the aircraft. Each of these actions has a required power to complete and a minimum operation time. Each of these actions are called K-conditions and are numbered from one to seven. Load profiles can then be built by putting a series of these blocks together one after another to form a full profile.

Each K number represents a specific action these are:

$\begin{array}{ll}\text { K1 } & \text { - Loading and Preparation } \\ \text { K2 } & \text { - Start and Warm-Up } \\ \text { K3 } & \text { - Taxi } \\ \text { K4 } & \text { - Take-Off and Climb } \\ \text { K5 } & \text { - Cruise } \\ \text { K6 } & \text { - Cruise/Combat } \\ \text { K7 } & \text { - Landing }\end{array}$

Table I shows the required current for each K-condition as a percentage of the generator maximum and has two values the first the 5 minute value represents the first 5 minutes of the action and is the start up power, while the continuous value is the load for the rest of the duration of the action. The initial load profile used in this test in shown in figure 8, this applies to both generators.

To avoid damaging the test rig these tests were carried out entirely in simulation using the models described earlier, both of which have been validated from it. In this case the

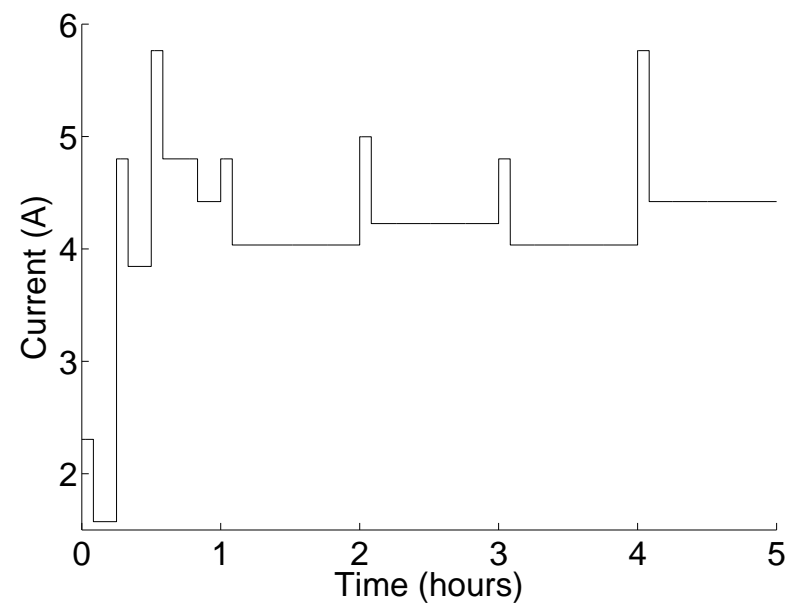

Fig. 8. Initial Load Profile.

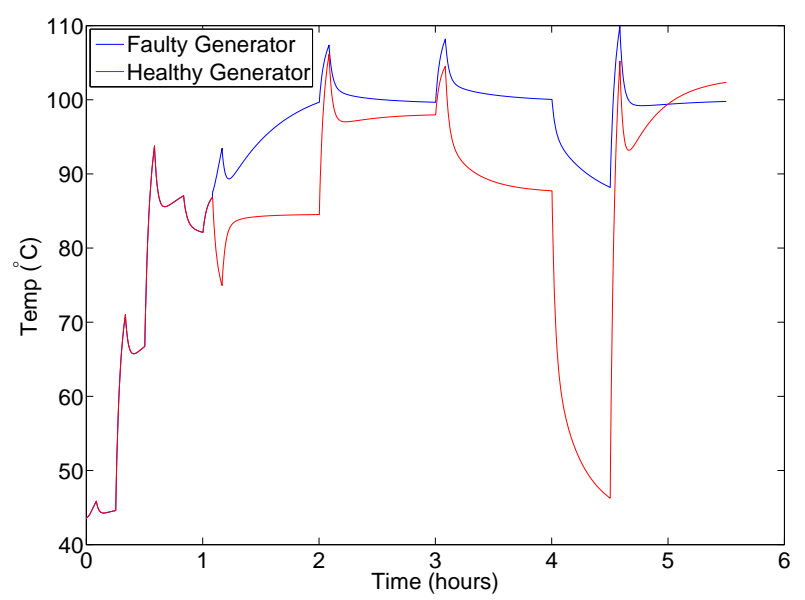

Fig. 9. Results showing actual winding temperature for both generators.

generators would be represented by the complex form of the model, referred to as the plant. The simplified model would be used as part of the Kalman Filter and for prediction purposes, referred to as the prediction model. This would test that the system could complete the load re-optimisation when nonperfect predictions are available as would be the base in an actual system on-board an aircraft.

For this test case one generator develops a fault, while the second remains healthy. The fault simulated is a coolant blockage after 67 minutes, this causes a reduction in mass flow through the system. it is assumed that the fault is detected meaning the prediction model is updated. The results are shown in figure 9 and 10.

Figures 9 and 10 show the stator iron and winding temperatures of both generators over time. In this case its not an issue if the temperature goes above the limit during the 5 minute start up period for a K-condition, however the temperature needs to quickly drop and stay below the limit during the continuous stage of K-condition. Figures 9 and 10 shows that 
TABLE I

K-CONDITONS

\begin{tabular}{c|c|c|c|c|c|c|c}
\hline & K1 & K2 & K3 & K4 & K5 & K6 & K7 \\
\hline \hline 5 mins & $40.0 \%$ & $83.3 \%$ & $100.0 \%$ & $83.3 \%$ & $83.3 \%$ & $86.7 \%$ & $100.0 \%$ \\
Continuous & $27.3 \%$ & $66.7 \%$ & $83.3 \%$ & $76.7 \%$ & $70.0 \%$ & $73.3 \%$ & $7 \%$ \\
\hline
\end{tabular}

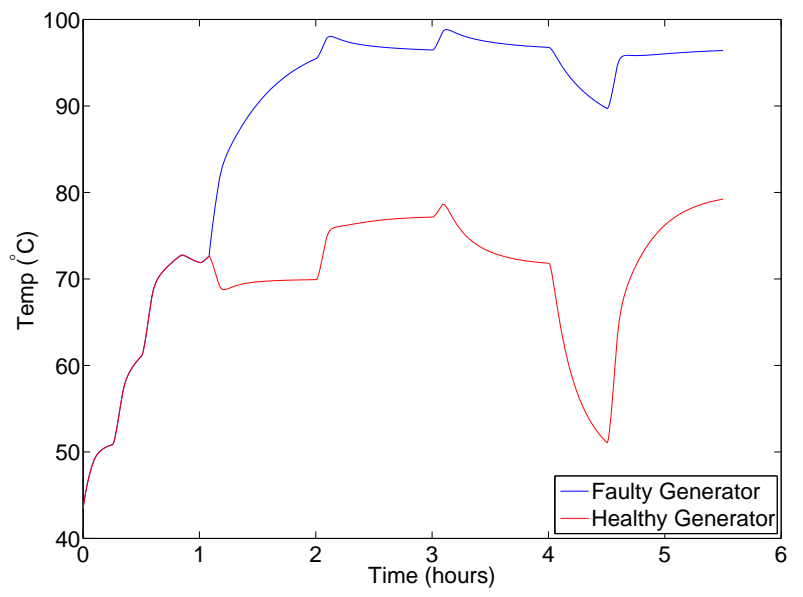

Fig. 10. Results showing actual stator iron temperature for both generators.

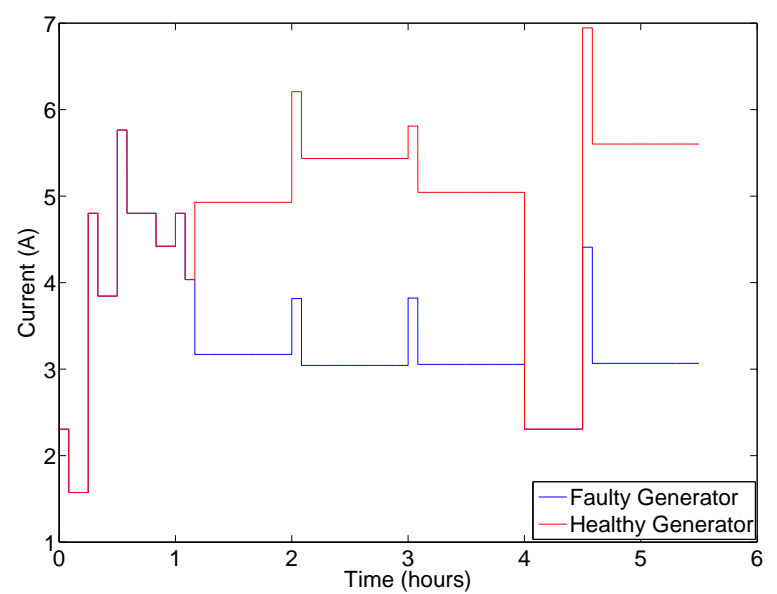

Fig. 11. The actual load profile applied to the generator over time after load management updates.

this is achieved by the system on both generators.

Figure 11 shows the current load of both generators over time. This graph clearly shows the action of the load management system and the steps taken to balance the load on the generator. Once the fault occurs the main action used is the displacement of the loads from the faulty to healthy generator. This is sufficient until four hours into the test where a rest period is required before the final action can be achieved.

\section{CONCLUSION}

A condition monitoring system capable of predicting and correcting overheat faults on aircraft generators has been shown. This was achieved by adjusting the load profiles of the on-board generators in flight when a thermal model of the generator predicts and overheat. The system shows the ability to create an acceptable solution while minimising the total mission length.

\section{REFERENCES}

[1] I. Moir, "More-electric aircraft-system considerations," IEE Colloquium on Electrical Machines and Systems for the More Electric Aircraft, 1999.

[2] J. Rosero, J. Ortega, E. Aldabas, and L. Romeral, "Moving towards a more electric aircraft," IEEE Aerospace and Electronic Systems Magazine, vol. 22, 2007.

[3] N. Iyer, K. Goebel, and P. Bonissone, "Framework for post-prognostic decision support," in Aerospace Conference, 2006 IEEE. IEEE, 2006, pp. $10-$ pp.

[4] A. Hess, G. Calvello, and T. Dabney, "Phm a key enabler for the jsf autonomic logistics support concept," in Aerospace Conference, 2004. Proceedings. 2004 IEEE, vol. 6. IEEE, 2004, pp. 3543-3550.

[5] V. Rouet, K. Moreau, and B. Foucher, "Embedded prognostics and health monitoring systems," in Electronics System-Integration Technology Conference, 2008. ESTC 2008. 2nd. IEEE, 2008, pp. 79-84.

[6] L. Tang, J. Ge et al., "Dynamic decision support and automated fault accommodation for jet engines," in 2007 IEEE Aerospace Conference, 2007, pp. 1-9.

[7] M. J. Watson, C. S. Byington, and A. Behbahani, "Very high frequency monitoring system for engine gearbox and generator health management," in Proceedings of the SAE AeroTech Congress \& Exhibition, no. 2007-01, 2007, p. 3878.

[8] T. D. Batzel and D. C. Swanson, "Prognostic health management of aircraft power generators," Aerospace and Electronic Systems, IEEE Transactions on, vol. 45, no. 2, pp. 473-482, 2009.

[9] J. Graham, R. Dixon, and K. Gregory, "Predicting the thermal state of generators on-board uavs," in SAE Technical Paper, 2013.

[10] P. Mellor, D. Roberts, and D. Turner, "Lumped parameter thermal model for electrical machines tefc design," in IEE Proceedings-B Electric Power Applications, vol. 138, 1991.

[11] I. Perez and J. Kassakian, "Stationary thermal-model for smooth air-gap rotating electric machines," Electrical Machines and Electromechanics, vol. 3, no. 3 - 4, 1979.

[12] G. Welch and G. Bishop, "An introduction to the kalman filter," 1995.

[13] R. G. Brown and P. Hwang, Introduction to Random Signals and Applied Kalman Filtering. New York: John Wiley \& Sons, 1997. 